\title{
The chance of a live birth after assisted reproduction in women with thyroid disorders
}

This article was published in the following Dove Press journal:

Clinical Epidemiology

\author{
Line Riis Jølving ${ }^{1,2}$ \\ Michael Due Larsen ${ }^{1,2}$ \\ Jens Fedder (iD) 3,4 \\ Sonia Friedman 1,2,5,6 \\ Bente Mertz Nørgård 1,2,5,6 \\ 'Center for Clinical Epidemiology, \\ Odense University Hospital, Odense, \\ Denmark; ${ }^{2}$ Research Unit of Clinical \\ Epidemiology, Department of Clinical \\ Research, University of Southern \\ Denmark, Odense, Denmark; ${ }^{3}$ Centre of \\ Andrology and Fertility Clinic, \\ Department D, Odense University \\ Hospital, Odense, Denmark; ${ }^{4}$ Research \\ Unit of Human Reproduction, \\ Department of Clinical Research, \\ University of Southern Denmark, \\ Odense, Denmark; ${ }^{5}$ Crohn's and Colitis \\ Center, Brigham and Women's Hospital, \\ Boston, MA, USA; ${ }^{6}$ Division of \\ Gastroenterology, Hepatology and \\ Endoscopy Research, Harvard Medical \\ School, Boston, MA, USA
}

Purpose: Women with thyroid disorders may have increased infertility and poor reproductive outcomes, but it is unclear if assisted reproductive technology (ART) is effective in this population. The aim of this study was to examine the chance of a pregnancy (biochemical and clinical), and a live birth after ART, in women with thyroid disorders undergoing ART treatment, compared to women without thyroid disorders. Among live-born children, we assessed the risk of congenital malformations.

Patients and methods: In a nationwide cohort study of all women undergoing ART treatments in Denmark from 1 January 1994 throughout June 2017, we calculated the chance of a pregnancy and a live birth after embryo transfer. Women with thyroid disorders were stratified into two groups: those diagnosed with hypothyroid or hyperthyroid disorders. The adjusted OR (aOR) of a biochemical and a clinical pregnancy, a live born child and a congenital malformation was computed using multilevel logistic regression models.

Results: In total, 199,674 embryo transfers were included in 2,101 women with thyroid disorders and in 65,526 women without thyroid disorders. The chance of a biochemical pregnancy was significantly reduced in women with hyperthyroidism $(\mathrm{aOR}=0.80,95 \% \mathrm{CI}$ 0.69-0.93), and the aOR of a live birth was $0.86,95 \%$ CI $0.76-0.98$. The aOR for a live birth in women with hypothyroidism was 1.03 (95\% CI 0.94-1.12). Children of women with hypothyroidism, who were conceived after ART treatment, had a significantly increased risk of any congenital malformation $(\mathrm{aOR}=1.46$ [95\% CI 1.07-2.00]).

Conclusion: Women with hyperthyroidism receiving ART treatment had a decreased chance of a live birth per embryo transfer compared to women without thyroid disorders. Women with hypothyroidism did not have a decreased chance of a live birth but their offspring had an increased risk of congenital malformation.

Keywords: assisted reproductive technology, in vitro fertilization, hyperthyroidism, hypothyroidism, clinical epidemiology

\section{Introduction}

Thyroid disorders are prevalent conditions in women of reproductive age ${ }^{1-4}$ and may affect female fertility in various ways, including anovulatory cycles, luteal phase defect, high prolactin levels, and sex hormone imbalances, ${ }^{5-7}$ which may cause both infertility and adverse pregnancy outcomes. ${ }^{8-12}$ Assisted reproductive technology (ART) treatment is a relevant intervention in women who cannot conceive naturally, and recent studies have shown that women with ulcerative colitis, Crohn's disease, and rheumatoid arthritis have a decreased chance of giving birth to a live-born child after ART treatment compared to other women undergoing ART treatment. ${ }^{13-15}$ All of these diseases share an autoimmune etiology. Thyroid disorders may also be autoimmune but the efficacy of ART treatment in women
Correspondence: Line Riis Jølving Center for Clinical Epidemiology, Odense University Hospital, Kløvervænget 30 entrance 216, Odense C DK-5000, Denmark

Tel +4524629638

Email line.joelving@rsyd.dk 
with thyroid disorders has never been investigated in a large cohort study. The main aim of our study was therefore to investigate ART outcomes in women with thyroid disorders, including hyperthyroidism and hypothyroidism.

Infertility is the failure to achieve a successful pregnancy after 12 months of regular unprotected intercourse. ${ }^{16}$ In general, up to $10-16 \%$ of all women suffer from infertility and many of them seek ART treatment, ${ }^{17-19}$ which includes in vitro fertilization (IVF), with or without fertilization with intracytoplasmic sperm injection (ICSI) and transfer of fresh, frozen-thawed (FER) embryos and vitrified-warmed blastocyst replacement (WBR), respectively. The impact of subclinical hypothyroidism, thyroid autoimmunity, and the medical treatment of thyroid disorders on the ART efficacy have been studied according to outcomes, such as miscarriage, clinical pregnancy, and live birth rates. ${ }^{16,20-29} \mathrm{~A}$ recent review from Busnelli et al further concludes that the presence of thyroid antibodies may have a detrimental effect on the course of a pregnancy determining an increased risk of miscarriage and a decreased chance of a live birth, but that age for instance may have impact on this. ${ }^{27}$ The efficacy of ART treatment in women with hyperthyroidism and hypothyroidism in terms of biochemical pregnancy, clinical pregnancy, and live birth has never been studied based on nationwide data. We aimed to examine the efficacy of ART treatment in women diagnosed with hyperthyroidism and hypothyroidism prior to ART treatment, compared to women without thyroid disorders using the Danish nationwide registers. The outcome was biochemical pregnancy, clinical pregnancy, and live birth. An association between female thyroid disorders and the risk of congenital malformation (CM) in the offspring has been described earlier, either as a consequence of the underlying maternal thyroid dysfunction or a teratogenic effect. ${ }^{4,30-32}$ No previous studies have examined if this is also the case if the pregnancy is ART induced, and we therefore aimed to estimate the risk of any CM in the live-born offspring.

\section{Materials and methods}

\section{Setting and the study population}

Data were obtained from the following nationwide Danish registries: 1) data on diagnoses in women with hyperthyroidism and hypothyroidism, and comorbidity from the Danish National Patient Register (DNPR), 2) data related to ART treatments, including the cause of infertility, and furthermore information on the achievement of a biochemical and clinical pregnancy were extracted from the Danish ART register, 3) data on the outcomes of live birth and
CMs from the ART register and the Danish Medical Birth Register (DMBR); and 4) civil registration number from the Civil Registration System (CRS), including vital status, emigration, and immigration. Data from all sources were linked at a personal level using the unique 10-digit civil registration number assigned at all Danish residents at birth or immigration from the CRS and used in all Danish health care registries and as linkage between registries. All data were encrypted so that no individuals could be identified by the researchers.

The Danish population is approximately 5.6 million inhabitants. The study population comprised all women with a valid civil registration number who were available for follow-up, initiating ART treatment with at least one embryo transfer during the study period of 1 January 1994 30 June 2017. In Denmark, all infertile couples and single women are offered up to three free fully reimbursed fresh fertility treatment attempts as long as the woman is younger than 41 years of age at the time of the referral to ART treatment. All ART-related treatment cycles are recorded in the Danish ART register. The register was established on 1 January 1994 and includes data on ART treatments from both public and private clinics ${ }^{33,34}$ and registration of all procedures and treatments is mandatory. In this study, ART refers to traditional IVF, ICSI, and FER/WBR.

\section{The exposed cohorts}

From the study population of all women receiving ART treatment during the study period, we identified all women with the diagnoses hyperthyroidism and hypothyroidism in the DNPR. The DNPR includes records of all patients discharged from Danish hospitals since 1977 and all outpatient visits since 1994. ${ }^{35,36}$ Information in the DNPR includes hospital, dates of admission, discharge, procedures, and diagnoses based on the ICD-8 (before 1994 and ICD-10 thereafter, the ICD-9 classification was never used in Denmark), and civil registration numbers. All eligible patients who had a discharge history, or outpatient visit, of hyperthyroidism from any hospital in Denmark (ICD-8 codes: 242.00-242.29; ICD-10 codes: E05-E05.9 [except E05.4, E05.8A, and E05.9A]) before the date of embryo transfer, were identified. ${ }^{37,38}$ Similarly, all eligible patients, who had a discharge history of hypothyroidism from any hospital in Denmark (ICD-8 codes: 243.99, 244-244.09 [except 244.02]; ICD-10 codes: E03-E03.9 [except E03.0A, E03.1B, and E03.4], E89.0) before the date of embryo transfer, were identified. ${ }^{37,38}$ Women registered with diagnoses in both categories (hyperthyroidism and 
hypothyroidism) were excluded from the analyses (468 transfers in 164 patients). For the outcomes biochemical pregnancy, clinical pregnancy and live birth, the exposed cohorts comprised all ART treatment cycles in women with hyperthyroidism or hypothyroidism, respectively. Since each woman could have several ART treatments, the observation unit was the embryo transfer. ${ }^{39-41}$ According to the outcome of CM, each woman with hyperthyroidism or hypothyroidism could have had several childbirths during the study period and the observational unit for examining the risk of CMs was the live-born child.

\section{The unexposed cohort}

For the outcomes of biochemical pregnancy, clinical pregnancy and live birth, the unexposed cohort comprised all embryo transfers in women without diagnoses of hyperthyroidism or hypothyroidism before the date of embryo transfer. For the outcome CMs, the unexposed cohort comprised all live-born children in the population of women without hyperthyroidism or hypothyroidism.

\section{Outcomes}

The outcome for the exposed and unexposed cohorts was live birth within a period of 140-308 days after the date of embryo transfer. We retrieved information regarding live birth after ART treatment from the DMBR. The DMBR includes information on all births in Denmark since 1 January 1973 and also includes information on the mother, the child, birth-related information, and diagnoses of CMs (from 2006). ${ }^{42-44}$ A live birth was thus considered to be the result of the particular ART treatment if the difference between embryo transfer and birth was 20-44 weeks (corresponding to $140-308$ days ${ }^{13,39}$ from the last menstruation start. The outcome biochemical pregnancy was based on information from the ART register by a positive human chorionic gonadotropin ( $\mathrm{hCG}$ ) measurement at 14-16 days after embryo transfer, and a clinical pregnancy was based on ultrasound examination approximately 7-8 weeks after embryo transfer. Complete information on clinical pregnancies, in terms of positive ultrasound examination, was available in the register from 2006. The outcome of any CM diagnosed within 1 year afterbirth was based on information from the DMBR (for CM diagnoses please refer to supplemental).

\section{Data on confounders}

The covariates included in the statistical analyses were selected a priori. From the DNPR we obtained data on comorbid diseases prior to each embryo transfer for all women in the study population and a comorbidity index score, the Charlson comorbidity index score, covering 19 major disease categories, weighted according to their prognostic impact, was conducted. ${ }^{45}$ Charlson comorbidity index is a validated tool to adjust case-mix and the most often used measurement for comorbidity in register-based studies. The overall positive predictive values for the 19 Charlson conditions were consistently high with $98.0 \%$ in a Danish validation study. ${ }^{46}$ The scores were calculated for each embryo transfer of each woman in two index levels: no comorbidity (Charlson index 0) and some comorbidity (Charlson index $\geq 1)$. Information on parity was obtained in two categories $(0,1+)$ and smoking in two categories (yes/no).

For the entire study period we had data on the woman's age at the time of embryo transfer (continuous variable), calendar year of the ART treatment in four periods (19941999, 2000-2005, 2006-2011, and 2012-2017), type of treatment (IVF, ICSI, FER/WBR), and the cause of infertility (female factor, male factor, combined female/male factor, and idiopathic infertility). From 2006, the ART register also contained information on the BMI in four categories corresponding to the WHO classification: underweight $\left(<18.5 \mathrm{~kg} / \mathrm{m}^{2}\right)$, normal weight $\left(18.5-24.9 \mathrm{~kg} / \mathrm{m}^{2}\right)$, overweight $\left(25.0-29.9 \mathrm{~kg} / \mathrm{m}^{2}\right)$ and obese $\left(\geq 30 \mathrm{~kg} / \mathrm{m}^{2}\right),{ }^{47}$ partners age, and alcohol intake at the time of embryo transfer in two categories (yes/no).

\section{Statistical analyses}

Contingency tables were constructed for the main study variables according to the two exposed cohorts, and the unexposed cohort. We used multilevel logistic regression analyses to compute the crude and the adjusted relative risk estimates as ORs with 95\% CIs for a biochemical pregnancy, clinical pregnancy and live births following ART treatments in women with hyperthyroidism or hypothyroidism relative to women without thyroid disorders. The model accounted for multiple embryo transfers in the same woman. Adjustments were made for Charlson comorbidity index, the women's age at the time of embryo transfer, calendar year of the ART treatment, the type of infertility treatment, and the cause of infertility. In an extended regression model, including data from 2006 and onwards, we further adjusted for BMI, partner's age, female smoking, and alcohol intake at the time of embryo transfer. These factors, however, did not change the estimates and were omitted from the final model.

We used logistic regression computing the crude and the adjusted relative risk estimates for CMs diagnosed in 
all live-born children in singleton pregnancies, conceived after ART treatment in women with hyperthyroidism compared to children conceived after ART in women without thyroid disorders, and likewise in women with hypothyroidism compared to children after ART in women without thyroid disorders. The model accounted for multiple children by the same woman. In the analyses of the risk of $\mathrm{CMs}$, we restricted our data to include only births after ART treatment between 1 January 2006 and 30 June 2016, in order to have information on CMs in the offspring from the DMBR and at least 1-year follow-up. Diagnoses of dislocations of the hip and undescended testis were excluded from CMs due to low validity of these registrations. $^{48}$

All data were stored and linked at a secure server, at the Danish health data authority (The "Sundhedsdatastyrelsen"). All statistical analyses were conducted using Stata V. 15.0 software (StataCorp LP, College Station, TX, USA).

\section{Approvals and ethical considerations}

The study is notified at the Danish Data Protection Agency under the current joint notification of the Region of Southern Denmark (ref.no. 2012-58-0018). According to Danish law, ethical review board approval or patient consent are not required for register-based studies.

\section{Results}

Table 1 provides the characteristics for all ART treatments in the two exposed cohorts and the unexposed cohort. A total of 2,010 embryo transfers in 698 women with hyperthyroidism, and 3,692 embryo transfers in 1,403 women with hypothyroidism conducted the exposed cohort during the study period of January 1 1994-30 June 2017. The unexposed cohort compromised a total of 193,972 embryo transfers in 65,526 women without thyroid disorders. In the two exposed cohorts, the cause of infertility was referred to as a female factor in $24.4 \%$ in hyperthyroidism, $13.2 \%$ in hypothyroidism, and $25.1 \%$ in the unexposed cohort. The frequency of ART treatments increased during the study period in all three cohorts. In women diagnosed with hyperthyroidism, $43.2 \%$ of the embryo transfers were in 2012-2017, compared to $76.5 \%$ in hypothyroidism, and $30.2 \%$ in the unexposed cohort during the same period. The median female age and the distribution according to smoking and alcohol consumption were similar in the unexposed and exposed cohorts whereas fewer women with hypothyroidism had normal weight compared to those with hyperthyroidism and the unexposed cohort. Furthermore, the proportion of obese women was $11.4 \%$ in women with hyperthyroidism, $12.1 \%$ in women with hypothyroidism, and $9.7 \%$ in the unexposed cohort. At the time of embryo transfer in patients with hyperthyroidism, the median duration of the thyroid disease was 52 months (25-75 percentiles: 21-98), and for patients with hypothyroidism the median duration of thyroid disease was 20 months (25-75 percentiles: 8-42). Women with hyperthyroidism and hypothyroidism had more comorbid diseases than the women without thyroid disorders. However, the vast majority of women in all cohorts had no comorbid disorders.

\section{Outcomes: biochemical and clinical pregnancy and live birth}

The chance of a biochemical pregnancy after each embryo transfer in women with hyperthyroidism and hypothyroidism is shown in Table 2, as is the chance of a clinical pregnancy. The adjusted OR of a positive hCG test after embryo transfer in women with hyperthyroidism was 0.80 (95\% CI 0.69-0.93), and the aOR of a clinical pregnancy detected by an ultrasound examination was also reduced, 0.74 (95\% CI 0.58-0.96).

In women with hypothyroidism undergoing ART treatment, the chance of a biochemical pregnancy and clinical pregnancy was not decreased, aOR $=1.05$ (95\% CI 0.97-1.14) and 0.95 (95\% CI $0.81-1.12)$, respectively.

A total of $19.3 \%$ of the women with hyperthyroidism had a live-born child after embryo transfer, and $24.1 \%$ of the women in the unexposed cohort. The ORs for live birth in women with hyperthyroidism, compared with women without thyroid disorders, are given in Table 3 . The chance of a live birth was significantly reduced for each embryo transfer in women with hyperthyroidism, aOR $0.86(95 \%$ CI $0.76-0.98)$. A total of $23.6 \%$ of the women with hypothyroidism had a live-born child after embryo transfer (Table 3), and the chance of a live birth after embryo transfer in women with hypothyroidism, compared to women without thyroid disorders was close to unity (aOR=1.03, 95\% CI 0.94-1.12).

\section{Congenital malformations}

Children of women with hyperthyroidism had no significantly increased risk of $\mathrm{CM}, \mathrm{aOR}=1.03(0.62-1.74)$ (Table 4). Children born to women with hypothyroidism had a significantly increased risk of CM compared to 


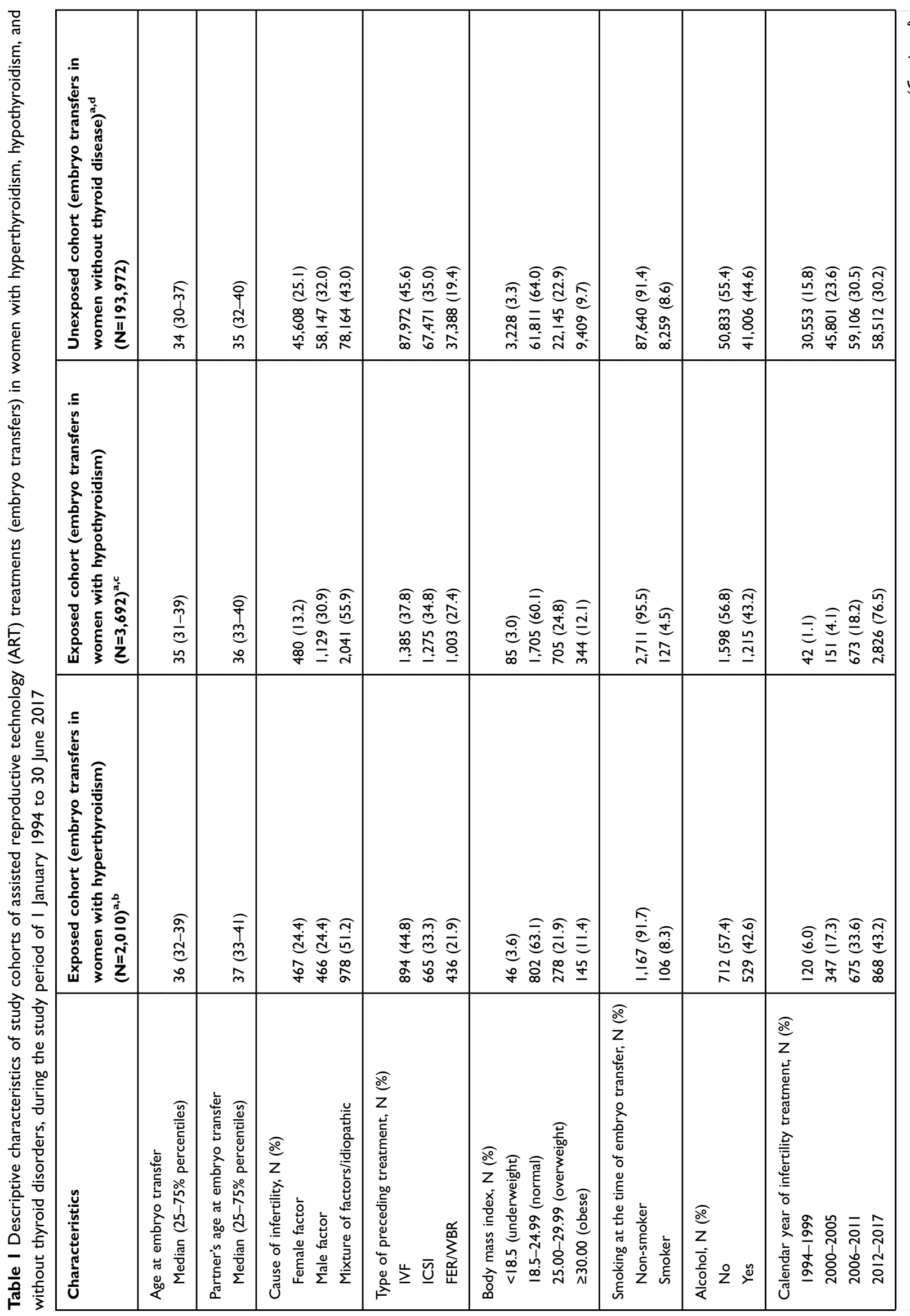




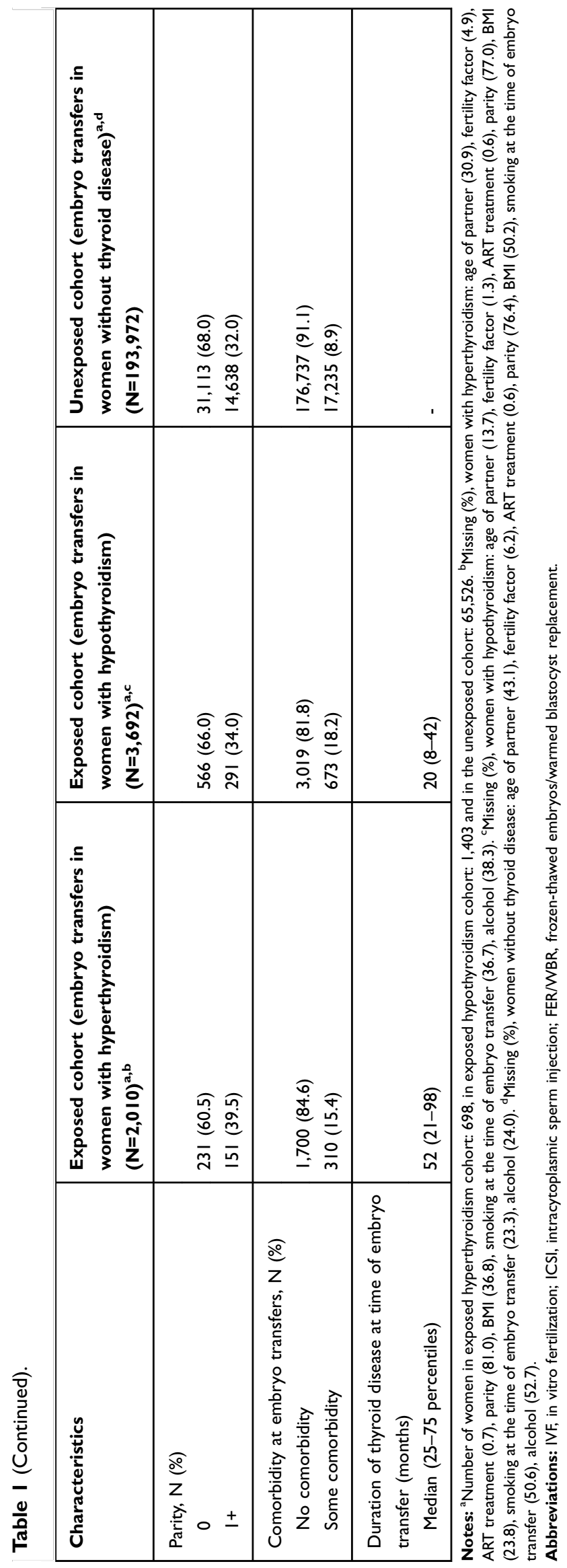

children of women without thyroid disorder, $\mathrm{aOR}=1.46$ (95\% CI 1.07-2.00). The types of CMs for women with hyperthyroidism and hypothyroidism (Table S1 and S2) for all singleton pregnancies during the restricted study period.

\section{Discussion}

In this nationwide cohort study of 23.5 years of assisted reproduction, we found that women with hyperthyroidism had a statistically significantly decreased chance of a live birth per embryo transfer, compared to embryo transfers in women without thyroid disorders. Our results also indicate that the reason for the decreased chance of a live birth could be related to inadequate implantation or early development of the embryo in the uterus, although the reasons so far are speculative. On the contrary, the chance of a live birth after embryo transfer in women with hypothyroidism was comparable to women without thyroid disorders undergoing ART treatment. Finally, our results suggest that children conceived through ART treatment in women with hypothyroidism had an increased risk of any $\mathrm{CM}$, compared to children where the mother did not have thyroid disorders or had hyperthyroidism.

Examining the efficacy of ART in women with thyroid disorders is relevant for a number of reasons. Firstly, the prevalence of women with thyroid disorders during their fertile years has increased during the past decades from $0.41 \%$ in $1989-1993$ to $2.29 \%$ in $2009-2013 .{ }^{1}$ Secondly, there is probably an association between thyroid autoimmunity and infertility. Studies have suggested an association between autoimmune thyroid disease and infertility; $\mathrm{RR}=2.1 \quad(95 \%$ CI $1.7-2.6) .{ }^{49}$ One small study showed a prevalence of infertility in women with hyperthyroidism of $5-8 \%,{ }^{50}$ whereas another study showed hypothyroidism in $9.4 \%$ of women with impaired fertility, ${ }^{51}$ and in women presenting with autoimmune dysthyroid, the prevalence of infertility reached 47\% (Graves' disease) and 52\% (Hashimotos' thyroiditis) with persistence of infertility despite obtaining euthyroid. ${ }^{52}$ These conclusions may also be relevant for women undergoing ART treatment. In a study of totally 2,012 women, 90 women presenting with anti-thyroid antibodies, in a cohort of 676 infertile women, had a significantly lower rate of fertilization, implementation, and pregnancy than women without these antibodies $64.3 \%$ vs $74.6 \%, 17.8 \%$ vs $27.1 \%$, and $33.3 \%$ vs $46.7 \%$, respectively. ${ }^{53}$

Thyroid hormones are essential developmental factors and perform their regulatory action by binding to nuclear 
Table 2 The chance of a biochemical pregnancy and a clinical pregnancy in women with hyperthyroidism and hypothyroidism after assisted reproductive technology (ART) treatments (embryo transfers), during the study period of I January 1994 to 30 June 2017

\begin{tabular}{|c|c|c|c|c|}
\hline & $\begin{array}{l}\text { Exposed cohort } \\
\text { (embryo transfers } \\
\text { in women with } \\
\text { hyperthyroidism) }\end{array}$ & $\begin{array}{l}\text { Unexposed cohort } \\
\text { (embryo transfers } \\
\text { in women without } \\
\text { thyroid disease) }\end{array}$ & Crude OR $(95 \% \mathrm{Cl})$ & $\begin{array}{l}\text { Adjusted OR } \\
(95 \% \mathrm{Cl})^{\mathrm{a}}\end{array}$ \\
\hline \multicolumn{5}{|l|}{ Biochemical pregnancy (hCG), ${ }^{b} \mathbf{N}(\%)$} \\
\hline Yes & $610(30.65)$ & $68,627(35.63)$ & $0.78(0.70-0.87)$ & $0.80(0.69-0.93)$ \\
\hline No & $1,380(69.35)$ & $123,992(64.37)$ & & \\
\hline \multicolumn{5}{|l|}{ Clinical pregnancy (ultrasound), ${ }^{c} \mathbf{N}(\%)$} \\
\hline Yes & $345(76.67)$ & $33.675(82,22)$ & $0.69(0.54-0.88)$ & $0.74(0.58-0.96)$ \\
\hline \multirow[t]{2}{*}{ No } & $105(23.33)$ & $7,280(17.78)$ & & \\
\hline & $\begin{array}{l}\text { Exposed cohort } \\
\text { (embryo transfers } \\
\text { in women with } \\
\text { hypothyroidism) }\end{array}$ & $\begin{array}{l}\text { Unexposed cohort } \\
\text { (embryo transfers } \\
\text { in women without } \\
\text { thyroid disease) }\end{array}$ & Crude OR $(95 \% \mathrm{Cl})$ & $\begin{array}{l}\text { Adjusted OR } \\
(95 \% \mathrm{Cl})^{\mathrm{a}}\end{array}$ \\
\hline \multicolumn{5}{|l|}{ Biochemical pregnancy (hCG), ${ }^{d} \mathbf{N}(\%)$} \\
\hline Yes & $1,318(36.48)$ & $68,627(35.63)$ & $1.05(0.97-1.13)$ & $1.05(0.97-1.14)$ \\
\hline No & $2,295(63.52)$ & I23,992 (64.37) & & \\
\hline \multicolumn{5}{|l|}{ Clinical pregnancy (ultrasound), ${ }^{\mathrm{e}} \mathbf{N}(\%)$} \\
\hline Yes & $\mathrm{I}, 012(8 \mathrm{I} .55)$ & $33,674(82.22)$ & $0.95(0.80-1.1 I)$ & $0.95(0.8 I-I .12)$ \\
\hline No & $229(18.45)$ & $7,28 \mid(17.78)$ & & \\
\hline
\end{tabular}

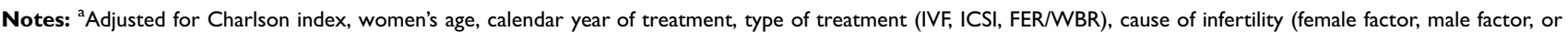
mixture of factors/idiopathic). ' Number of embryo transfers in the exposed cohort: 1,990 (number of women: 697, number of missing: 20). Number of embryo transfers in the unexposed cohort: 192,619 (number of women: 65,486, number of missing: 1,353). 'Restricted to 2006 -June 2017 due to only valid data on ultrasound from 2006. Number of embryo transfers in the exposed cohort: 450 (number of women: 328) missing/not measured: I,093. Number of embryo transfers in the unexposed cohort: 40,955 (number of women: 28,364) missing/not measured: 76,663. 'Number of embryo transfers in the exposed cohort: 3,613 (number of women: I,399, number of missing:

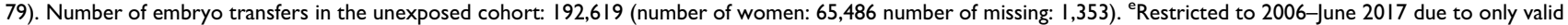
data on ultrasound from 2006. Number of embryo transfers in the exposed cohort: I,24I (number of women: 3,499) missing/not measured: 2,213 . Number of embryo transfers in the unexposed cohort: 40,955 (number of women: 28,364 ) missing/not measured: 76,663.

Table 3 The chance of live birth in women with hyperthyroidism and hypothyroidism after assisted reproductive technology (ART) treatments (embryo transfers), during the study period of I January 1994-30 June 2017

\begin{tabular}{|c|c|c|c|c|}
\hline & $\begin{array}{l}\text { Exposed cohort (embryo } \\
\text { transfers in women with } \\
\text { hyperthyroidism) } \\
\left(\mathrm{N}=2,0(0)^{\mathrm{a}}\right.\end{array}$ & $\begin{array}{l}\text { Unexposed cohort (embryo } \\
\text { transfers in women without } \\
\text { thyroid disease) }(\mathrm{N}=193,972)^{b}\end{array}$ & Crude OR $(95 \% \mathrm{Cl})$ & Adjusted OR ${ }^{c}(95 \% \mathrm{Cl})$ \\
\hline \multicolumn{5}{|l|}{ Live birth, $\mathbf{N}(\%)$} \\
\hline Yes & $388(19.30)$ & $46,716(24.08)$ & $0.74(0.65-0.84)$ & $0.86(0.76-0.98)$ \\
\hline \multirow[t]{2}{*}{ No } & I,622 (80.70) & $147,256(75.92)$ & & \\
\hline & $\begin{array}{l}\text { Exposed cohort (embryo } \\
\text { transfers in women with } \\
\text { hypothyroidism) } \\
(\mathrm{N}=3,692)^{d}\end{array}$ & $\begin{array}{l}\text { Unexposed cohort (embryo } \\
\text { transfers in women without } \\
\text { thyroid disease) }(\mathrm{N}=\mid 93,972)^{\mathrm{e}}\end{array}$ & Crude OR $(95 \% \mathrm{Cl})$ & Adjusted OR ${ }^{c}(95 \% \mathrm{Cl})$ \\
\hline \multicolumn{5}{|l|}{ Live birth } \\
\hline Yes & $870(23.56)$ & $46,716(24.08)$ & $0.99(0.90-1.08)$ & $1.03(0.94-1.12)$ \\
\hline No & $2,822(76.44)$ & 147,256 (75.92) & & \\
\hline
\end{tabular}

Notes: ${ }^{2}$ Number of women in the exposed cohort: 698 . ${ }^{\mathrm{b}}$ Number of women in the unexposed cohort: 65,526 . 'Adjusted for Charlson index, women's age, calendar year of treatment, type of treatment (IVF, ICSI, FER/WBR), cause of infertility (female factor, male factor, or mixture of factors/idiopathic). ${ }^{\mathrm{d}} \mathrm{Number}$ of women in the exposed cohort: I,403. ${ }^{\mathrm{e}}$ Number of women in the unexposed cohort: $65,526$. 
Table 4 Crude and adjusted ORs, with $95 \% \mathrm{Cl}$, for congenital malformations (CMs) in live born children, conceived with assisted reproductive technology (ART) treatments of women with hyperthyroidism and hypothyroidism, compared with children born to women without thyroid disease. Study period from I January 2006 to 30 June 2016, and followed until 30 June 2017

\begin{tabular}{|c|c|c|c|c|}
\hline & $\begin{array}{l}\text { Exposed cohort (live } \\
\text { born by women with } \\
\text { hyperthyroidism) }^{a}\end{array}$ & $\begin{array}{l}\text { Unexposed cohort (live } \\
\text { born by women without } \\
\text { thyroid disease) }\end{array}$ & Crude OR $(95 \% \mathrm{Cl})$ & $\begin{array}{l}\text { Adjusted } O^{c}{ }^{c} \\
(95 \% \mathrm{Cl})\end{array}$ \\
\hline \multicolumn{5}{|l|}{ Congenital malformations, $\mathbf{N}(\%)$} \\
\hline Yes & $25(8.33)$ & $2,183(7.70)$ & I.II (0.68-1.82) & $1.03(0.62-1.74)$ \\
\hline \multirow[t]{2}{*}{ No } & $275(91.67)$ & $26,157(92.30)$ & & \\
\hline & $\begin{array}{l}\text { Exposed cohort (live } \\
\text { born by women with } \\
\text { hypothyroidism) }^{d}\end{array}$ & $\begin{array}{l}\text { Unexposed cohort (live } \\
\text { born by women without } \\
\text { thyroid disease) }^{\mathbf{e}}\end{array}$ & Crude OR $(95 \% \mathrm{Cl})$ & $\begin{array}{l}\text { Adjusted } \text { OR }^{c} \\
(95 \% \mathrm{Cl})\end{array}$ \\
\hline \multicolumn{5}{|l|}{ Congenital malformations, $\mathbf{N}(\%)$} \\
\hline Yes & $79(11.32)$ & $2,183(7.70)$ & $1.65(1.23-2.22)$ & $1.46(1.07-2.00)$ \\
\hline No & $619(88.68)$ & $26,159(92.30)$ & & \\
\hline
\end{tabular}

Notes: ${ }^{a}$ Number of exposed observations, live-born children (mothers): 300 (235). ' Number of non-exposed observations, live-born children (mothers): 28,340 (2I,493). 'Adjusted for Charlson index, women's age, calendar year of treatment, type of treatment (IVF, ICSI, FER/WBR), cause of infertility (female factor, male factor, or mixture of factors/idiopathic) and smoking at the time of embryo transfer. ${ }^{\mathrm{d}}$ Number of exposed observations, live-born children (mothers): 698 (574). ${ }^{\mathrm{e}} \mathrm{Number}$ of non-exposed observations, live-born children (mothers): $28,342(21,494)$

thyroid hormone receptors, and dysfunction may interfere with numerous aspects of reproduction, including maturation of the oocytes and menstrual cycle, ${ }^{54}$ stages of fertilization, implantation, and the ability to carry the pregnancy throughout all trimesters, ${ }^{55}$ and the presence of thyroid auto-antibodies is greater in the context of early ovarian insufficiency. ${ }^{56}$ Thirdly, results on the efficacy of ART treatment in women with other autoimmune diseases have suggested a reduced chance of a live birth after ART treatment in women with ulcerative colitis and Crohn's disease $(\mathrm{aOR}=0.78$ [95\% CI $0.67-0.91]$ and 0.61 [95\% CI 0.47-0.79], respectively), ${ }^{13,14}$ and 0.78 (95\% CI $0.56-0.92)$ in rheumatoid arthritis, ${ }^{15}$ though the underlying mechanisms are unknown.

In Denmark, all female infertility patients have routinely been screened for thyroid disorders with measurements of thyroid-stimulating hormone (TSH) levels at all fertility clinics during the past 5-10 years, ${ }^{57}$ and relevant medication is introduced prior to the infertility treatment. Emphasis has been placed on changes in the thyroid function and the consequences of thyroid disorders during pregnancy in the past decades. ${ }^{16,49}$ But, to the best of our knowledge, no previous nationwide cohort study has examined the chance of a live birth in women with hyperthyroidism and hypothyroidism, compared to women without thyroid dysfunction undergoing ART treatment. ${ }^{58}$ However, a number of studies have examined the impact of thyroid dysfunction on ART treatment, based on clinical biomarkers in terms of measured antibodies, follicle counts, TSH levels, and hormone treatment. ${ }^{6,20-22,24-28}$ In women with thyroid antibodies, there was no difference in the chance of pregnancy and live birth compared to euthyroid women, ${ }^{24-28}$ and identical results were presented in a meta-analysis of ART outcomes in women with different preconception TSH levels. ${ }^{59}$ The comparison of these data to ours is problematic because of different inclusions, ${ }^{16}$ but indications of an decreased chance of a live birth have been suggested. ${ }^{27}$ In our study, we examined hyperthyroidism and hypothyroidism separately.

A possible association between hyperthyroidism and hypothyroidism during pregnancy and the risk of different $\mathrm{CMs}$ in the offspring has been described earlier, ${ }^{30,60-62}$ either as a consequence of hormonal imbalances or as a teratogenic effect of anti-thyroid medication in mature pregnancy. Nevertheless, no previous studies have examined if an association between hyperthyroidism and hypothyroidism and CMs persists in women undergoing ART treatment. We confirmed a potential association between maternal hypothyroidism and $\mathrm{CM}$, but not in children born to women with hyperthyroidism which is in accordance with a group of untreated patients with Graves' disease in a study from Japan, ${ }^{62}$ but in contrast to findings referred to in a review from Cooper and Laurberg. ${ }^{61}$ However, none of the studies were on ART-treated women and our outcomes of CMs in both groups and restricted power of each type of anomalies are limited and the estimates should be interpreted with caution.

The precision and the validity of our study depends on the size of the study population, accurate classification of 
the exposure, the quality of the outcome data, and the ability to take into account the influence of important confounders. According to these aspects, our study has several strengths. Firstly, this study is based on a nationwide study population of all Danish women with hyperthyroidism and hypothyroidism receiving ART treatment and using all other ART-treated women as controls. This was possible due to the ART register, which is based on mandatory registration of all individual treatment cycles in private and public clinics. ${ }^{33,34}$ Secondly, the nature of the longlasting Danish health registries allowed a 23.5-year study period and the large cohort of ART treatments provided us with solid statistical power. Thirdly, information from the used Danish health registers is based on mandatory registrations and this produces data of high completeness and validity with a limited number of pitfalls. ${ }^{34-36,42-44}$ Regarding our outcome measurements on live birth, data from the DMBR are of high validity and completeness, and all new-born children are registered in this register. ${ }^{42-44}$ Furthermore, validation of the diagnoses of thyroid disorders in the general population only revealed misclassification in $2 \%$ of the cases in a study from $2002^{63}$ which is in accordance with the generally high validity of the register. ${ }^{35}$ Fourthly, we also had access to data on a number of relevant confounders, including the Charlson comorbidity index, information on the origin of the infertility in terms of male, female or idiopathic factor as important variables, ${ }^{58,64}$ type of ART treatment, calendar year of ART treatment, age, BMI, smoking at the time of embryo transfer, and alcohol intake at time of infertility treatment, and the partner's age at the time of infertility treatment. We only included women in the exposed cohort if the diagnoses were given before the embryo transfer, and to avoid misclassification; women who had both diagnoses of hyperthyroidism and hypothyroidism before embryo transfer were excluded. Finally, the outcomes studied were obtained independently of the hypotheses studied and exposure measurements, preventing differential misclassification of the outcome measurements.

The current study, however, also has limitations. We know from previous studies that biomarkers, including thyroid antibodies, TSH, thyroxine, etc. may play an important role on reproductive outcomes. However, we had no opportunity to examine a potential impact of biomarkers based in our dataset. Nonetheless, the TSH screening in infertile women introduces medical treatment if the levels are abnormal, and one might argue that mechanisms other than hormone imbalances contribute to the decreased chance of a live birth in our study. For instance, autoimmune factors could contribute to the decreased efficacy of ART if the underlying thyroid disorder is Graves' disease or Hashimoto's thyroiditis. ${ }^{58}$ We did not have information on the etiology of the thyroid disorder, and some women might have had a non-autoimmune etiology. We did not have information on disease activity or any treatment the women received for their thyroid disorders prior to the embryo transfer. Furthermore, our data do not provide evidence for the underlying reason for the $\mathrm{CM}$ although any potential causes might be multifarious. Finally, we only included women who had diagnoses of either hyperthyroidism or hypothyroidism before embryo transfer. We cannot rule out a potential misclassification of the thyroid disorders but any misclassification would most probably be nondifferential and bias our results toward conservative estimates. Another limitation is that we were unable to assess very early abortions and stillbirths due to CM, including those due to antenatal diagnoses of CMs and additionally the included diagnoses cover a wide span of CMs across different organ systems. It is also a limitation that we did not have sufficient statistical power to estimate the risk of CMs according to specific subtypes of CMs. Thus, we could have underestimated the true relative risk of $\mathrm{CM}$, if CMs after ART treatment resulting in stillbirths or very early abortions are more prevalent in women with hypothyroidism compared to women without thyroid disorders.

\section{Conclusion}

This study suggests that women with hyperthyroidism have a decreased chance of a live birth after ART treatment compared to women without thyroid disorders. Our results suggest that the cause could be related to an impaired chance of a biochemical and clinical pregnancy. These results also suggest that children, conceived through ART treatment in women with hypothyroidism, have an increased risk of CM diagnosed within their first year of life, but we cannot determine whether this finding is related to hypothyroidism or factors closely related to the disease (for instance medical treatments). Based on a nationwide cohort of ART treatments, these are the first results on the efficacy of ART treatment in women with hyperthyroidism and hypothyroidism. Future studies are warranted to confirm our findings and to understand the mechanisms behind the decreased chance of a live birth per embryo transfer in women with hyperthyroidism. It would be most valuable if 
such studies could include information on disease severity, medications, thyroid hormone levels, and antibodies.

\section{Acknowledgment}

The study was supported by the Beckett Foundation, Denmark, and Odense University Hospital.

\section{Author contributions}

All authors made substantial contributions to conception and design, acquisition of data, or analysis and interpretation of data; took part in drafting the article or revising it critically for important intellectual content; gave final approval of the version to be published; and agree to be accountable for all aspects of the work.

\section{Disclosure}

The authors report no conflicts of interest in this work.

\section{References}

1. Jolving LR, Nielsen J, Kesmodel US, Nielsen RG, Beck-Nielsen SS, Norgard BM. Prevalence of maternal chronic diseases during pregnancy - a nationwide population based study from 1989 to 2013. Acta Obstet Gynecol Scand. 2016;95(11):1295-1304. doi:10.1111/aogs.13007

2. Wang C, Crapo LM. The epidemiology of thyroid disease and implications for screening. Endocrinol Metab Clin North Am. 1997;26 (1):189-218. doi:10.1016/S0889-8529(05)70240-1

3. Andersen SL, Olsen J, Laurberg P. Maternal thyroid disease in the Danish National Birth Cohort: prevalence and risk factors. Eur $J$ Endocrinol. 2016;174(2):203-212. doi:10.1530/EJE-15-0640

4. Howley MM, Fisher SC, Van Zutphen AR, et al. Thyroid medication use and birth defects in the National Birth Defects Prevention Study. Birth Defects Res. 2017;109(18):1471-1481. doi:10.1002/bdr2.1095

5. Verma I, Sood R, Juneja S, Kaur S. Prevalence of hypothyroidism in infertile women and evaluation of response of treatment for hypothyroidism on infertility. Int J Appl Basic Med Res. 2012;2(1):17-19. doi:10.4103/2229-516X.96795

6. Korevaar TIM, Minguez-Alarcon L, Messerlian C, et al. Association of thyroid function and autoimmunity with ovarian reserve in women seeking infertility care. Thyroid. 2018;28(10):1349-1358. doi:10.1089/ thy.2017.0582

7. Cho MK. Thyroid dysfunction and subfertility. Clin Exp Reprod Med. 2015;42(4):131-135. doi:10.5653/cerm.2015.42.4.131

8. Twig G, Shina A, Amital H, Shoenfeld Y. Pathogenesis of infertility and recurrent pregnancy loss in thyroid autoimmunity. J Autoimmun. 2012;38(2-3):J275-281. doi:10.1016/j.jaut.2012.02.002

9. Artini PG, Uccelli A, Papini F, et al. Infertility and pregnancy loss in euthyroid women with thyroid autoimmunity. Gynecol Endocrinol. 2013;29(1):36-41. doi:10.3109/09513590.2012.705391

10. Practice Committee of the American Society for Reproductive M. Subclinical hypothyroidism in the infertile female population: a guideline. Fertil Steril. 2015;104(3):545-553. doi:10.1016/j. fertnstert.2015.05.028

11. Casey BM, Dashe JS, Wells CE, et al. Subclinical hypothyroidism and pregnancy outcomes. Obstet Gynecol. 2005;105(2):239-245. doi:10.1097/01.AOG.0000159040.51773.bf

12. Andersen SL, Olsen J, Laurberg P. Hypothyroidism and pregnancy loss: comparison with hyperthyroidism and diabetes in a Danish population-based study. Clin Endocrinol (Oxf). 2016;85(6):962-970. doi:10.1111/cen.13136
13. Friedman S, Larsen PV, Fedder J, Norgard BM. The reduced chance of a live birth in women with IBD receiving assisted reproduction is due to a failure to achieve a clinical pregnancy. Gut. 2017;66(3):556558. doi:10.1136/gutjnl-2016-311805

14. Norgard BM, Larsen PV, Fedder J, de Silva PS, Larsen MD, Friedman S. Live birth and adverse birth outcomes in women with ulcerative colitis and Crohn's disease receiving assisted reproduction: a 20-year nationwide cohort study. Gut. 2016;65(5):767-776. doi:10.1136/gutjnl-2015-311246

15. Norgard BM, Larsen MD, Friedman S, Knudsen T, Fedder J. Decreased chance of a live born child in women with rheumatoid arthritis after assisted reproduction treatment: a nationwide cohort study. Ann Rheum Dis. 2019;78(3):328-334. doi:10.1136/annrheumdis-2018-214619

16. De Leo S, Pearce EN. Autoimmune thyroid disease during pregnancy. Lancet Diabetes Endocrinol. 2018;6(7):575-586. doi:10.1016/S2213-8587(17)30402-3

17. Rachootin P, Olsen J. Prevalence and socioeconomic correlates of subfecundity and spontaneous abortion in Denmark. Int J Epidemiol. 1982;11(3):245-249. doi:10.1093/ije/11.3.245

18. Schmidt L. Psychosocial burden of infertility and assisted reproduction. Lancet. 2006;367(9508):379-380. doi:10.1016/S0140-6736(06) 68117-8

19. Mosher WD, Pratt WF. Fecundity and infertility in the United States: incidence and trends. Fertil Steril. 1991;56(2):192-193.

20. Cai Y, Zhong L, Guan J, et al. Outcome of in vitro fertilization in women with subclinical hypothyroidism. Reprod Biol Endocrinol. 2017;15(1):39. doi:10.1186/s12958-017-0257-2

21. Kim CH, Ahn JW, Kang SP, Kim SH, Chae HD, Kang BM. Effect of levothyroxine treatment on in vitro fertilization and pregnancy outcome in infertile women with subclinical hypothyroidism undergoing in vitro fertilization/intracytoplasmic sperm injection. Fertil Steril. 2011;95(5):1650-1654. doi:10.1016/j.fertnstert.2010.12.004

22. Abdel Rahman AH, Aly Abbassy H, Abbassy AA. Improved in vitro fertilization outcomes after treatment of subclinical hypothyroidism in infertile women. Endocr Pract. 2010;16(5):792-797. doi:10.4158/ EP09365.OR

23. Unuane D, Velkeniers B, Bravenboer B, et al. Impact of thyroid autoimmunity in euthyroid women on live birth rate after IUI. Hum Reprod. 2017;32(4):915-922.

24. Lukaszuk K, Kunicki M, Kulwikowska P, et al. The impact of the presence of antithyroid antibodies on pregnancy outcome following intracytoplasmatic sperm injection-ICSI and embryo transfer in women with normal thyreotropine levels. $J$ Endocrinol Invest. 2015;38(12):1335-1343. doi:10.1007/s40618-015-0377-5

25. Tan S, Dieterle S, Pechlavanis S, Janssen OE, Fuhrer D. Thyroid autoantibodies per se do not impair intracytoplasmic sperm injection outcome in euthyroid healthy women. Eur $J$ Endocrinol. 2014;170 (4):495-500. doi:10.1530/EJE-13-0790

26. Sakar MN, Unal A, Atay AE, et al. Is there an effect of thyroid autoimmunity on the outcomes of assisted reproduction? J Obstet Gynaecol. 2016;36(2):213-217. doi:10.3109/01443615.2015.1049253

27. Busnelli A, Paffoni A, Fedele L, Somigliana E. The impact of thyroid autoimmunity on IVF/ICSI outcome: a systematic review and metaanalysis. Hum Reprod Update. 2016;22(6):775-790. doi:10.1093/ humupd/dmw019

28. Toulis KA, Goulis DG, Venetis CA, et al. Risk of spontaneous miscarriage in euthyroid women with thyroid autoimmunity undergoing IVF: a meta-analysis. Eur J Endocrinol. 2010;162(4):643-652. doi:10.1530/EJE-09-0850

29. van Den Boogaard E, Vissenberg R, Land JA, et al. Significance of (sub)clinical thyroid dysfunction and thyroid autoimmunity before conception and in early pregnancy: a systematic review. Hum Reprod Update. 2011;17(5):605-619. doi:10.1093/humupd/dmr024

30. Kallen B, Norstedt Wikner B. Maternal hypothyroidism in early pregnancy and infant structural congenital malformations. J Thyroid Res. 2014;2014:160780. doi:10.1155/2014/160780 
31. Grattan MJ, Thomas DS, Hornberger LK, Hamilton RM, Midodzi WK, Vohra S. Maternal hypothyroidism may be associated with CHD in offspring. Cardiol Young. 2015;25(7):1247-1253. doi:10.1017/ S1047951114001887

32. Eshkoli T, Wainstock T, Sheiner E, Beharier O, Fraenkel M, Walfisch A. Maternal hypothyroidism during pregnancy and the risk of pediatric endocrine morbidity in the offspring. Am J Perinatol. 2018. Epub 2018 Nov 26.

33. Westergaard HB, Johansen AM, Erb K, Andersen AN. Danish National IVF Registry 1994 and 1995. Treatment, pregnancy outcome and complications during pregnancy. Acta Obstet Gynecol Scand. 2000;79(5):384-389. doi:10.1080/j.1600-0412.2000.079005384.x

34. Andersen AN, Westergaard HB, Olsen J. The Danish in vitro fertilisation (IVF) register. Dan Med Bull. 1999;46(4):357-360.

35. Schmidt M, Schmidt SA, Sandegaard JL, Ehrenstein V, Pedersen L, Sorensen HT. The Danish National Patient Registry: a review of content, data quality, and research potential. Clin Epidemiol. 2015;7:449-490. doi:10.2147/CLEP.S91125

36. Lynge E, Sandegaard JL, Rebolj M. The Danish National Patient Register. Scand J Public Health. 2011;39(7 Suppl):30-33. doi:10.1177/1403494811401482

37. Andersen SL, Laurberg P, Wu CS, Olsen J. Maternal thyroid dysfunction and risk of seizure in the child: a Danish nationwide cohort study. J Pregnancy. 2013;2013:636705. doi:10.1155/2013/636705

38. Andersen SL, Olsen J, Wu CS, Laurberg P. Smoking reduces the risk of hypothyroidism and increases the risk of hyperthyroidism: evidence from 450,842 mothers giving birth in Denmark. Clin Endocrinol (Oxf). 2014;80(2):307-314. doi:10.1111/cen.12279

39. Petersen GL, Schmidt L, Pinborg A, Kamper-Jorgensen M. The influence of female and male body mass index on live births after assisted reproductive technology treatment: a nationwide registerbased cohort study. Fertil Steril. 2013;99(6):1654-1662. doi:10.1016/j.fertnstert.2012.09.009

40. Fedder J, Loft A, Parner ET, Rasmussen S, Pinborg A. Neonatal outcome and congenital malformations in children born after ICSI with testicular or epididymal sperm: a controlled national cohort study. Hum Reprod. 2013;28(1):230-240. doi:10.1093/humrep/det071

41. Stern JE, Brown MB, Wantman E, Kalra SK, Luke B. Live birth rates and birth outcomes by diagnosis using linked cycles from the SART CORS database. J Assist Reprod Genet. 2013;30(11):1445-1450. doi:10.1007/s10815-013-0092-0

42. Bliddal M, Broe A, Pottegard A, Olsen J, Langhoff-Roos J. The Danish Medical Birth Register. Eur J Epidemiol. 2018;33(1):27-36. doi:10.1007/s10654-018-0356-1

43. Knudsen LB, Olsen J. The Danish Medical Birth Registry. Dan Med Bull. 1998;45(3):320-323.

44. Kristensen J, Langhoff-Roos J, Skovgaard LT, Kristensen FB. Validation of the Danish Birth Registration. J Clin Epidemiol. 1996;49(8):893-897. doi:10.1016/0895-4356(96)00018-2

45. Charlson ME, Pompei P, Ales KL, MacKenzie CR. A new method of classifying prognostic comorbidity in longitudinal studies: development and validation. J Chronic Dis. 1987;40(5):373-383. doi:10.1016/0021-9681(87)90171-8

46. Thygesen SK, Christiansen CF, Christensen S, Lash TL, Sorensen HT. The predictive value of ICD-10 diagnostic coding used to assess Charlson comorbidity index conditions in the population-based Danish National Registry of Patients. BMC Med Res Methodol. 2011;11:83. doi:10.1186/1471-2288-11-83
47. World Health Organization. Obesity: Preventing and Managing the Global Epidemic. Geneva: World Health Organization; 2000.

48. Larsen H, Nielsen GL, Bendsen J, Flint C, Olsen J, Sorensen HT. Predictive value and completeness of the registration of congenital abnormalities in three Danish population-based registries. Scand J Public Health. 2003;31(1):12-16. doi:10.1080/14034940210165163

49. Poppe K, Velkeniers B, Glinoer D. Thyroid disease and female reproduction. Clin Endocrinol (Oxf). 2007;66(3):309-321. doi:10.1111/j.1365-2265.2007.02752.x

50. Joshi JV, Bhandarkar SD, Chadha M, Balaiah D, Shah R. Menstrual irregularities and lactation failure may precede thyroid dysfunction or goitre. J Postgrad Med. 1993;39(3):137-141.

51. Feldthusen AD, Pedersen PL, Larsen J, Toft Kristensen T, Ellervik C, Kvetny J. Impaired fertility associated with subclinical hypothyroidism and thyroid autoimmunity: the Danish General Suburban Population Study. J Pregnancy. 2015;2015:132718. doi:10.1155/2015/132718

52. Abalovich M, Mitelberg L, Allami C, et al. Subclinical hypothyroidism and thyroid autoimmunity in women with infertility. Gynecol Endocrinol. 2007;23(5):279-283. doi:10.1080/09513590701259542

53. Zhong YP, Ying Y, Wu HT, et al. Relationship between antithyroid antibody and pregnancy outcome following in vitro fertilization and embryo transfer. Int J Med Sci. 2012;9(2):121-125. doi:10.7150/ijms.3467

54. Forhead AJ, Fowden AL. Thyroid hormones in fetal growth and prepartum maturation. $J$ Endocrinol. 2014;221(3):R87-R103. doi:10.1530/JOE-14-0025

55. Allan WC, Haddow JE, Palomaki GE, et al. Maternal thyroid deficiency and pregnancy complications: implications for population screening. $J$ Med Screen. 2000;7(3):127-130. doi:10.1136/jms.7.3.127

56. Poppe K, Glinoer D, Tournaye H, et al. Thyroid autoimmunity and female infertility. Verh K Acad Geneeskd Belg. 2006;68(5-6):357-377.

57. Hanna Kristina Bertoli CSSF, Hedeland RL, Klajnbard A, Larsen EC, Wetterstrand V, Wissing ML. Udredning af kvinden forud for fertilitetsbehandling. Dansk Fertilitetsselskab. 2018:1-31.

58. Simopoulou M, Sfakianoudis K, Maziotis E, et al. The impact of autoantibodies on IVF treatment and outcome: a systematic review. Int J Mol Sci. 2019;20(4):892. doi:10.3390/ijms20040892

59. Zhao T, Chen BM, Zhao XM, Shan ZY. Meta-analysis of ART outcomes in women with different preconception TSH levels. Reprod Biol Endocrinol. 2018;16(1):111. doi:10.1186/s12958-018-0362-x

60. Liu S, Joseph KS, Lisonkova S, et al. Association between maternal chronic conditions and congenital heart defects: a population-based cohort study. Circulation. 2013;128(6):583-589. doi:10.1161/ CIRCULATIONAHA.112.001054

61. Cooper DS, Laurberg P. Hyperthyroidism in pregnancy. Lancet Diabetes Endocrinol. 2013;1(3):238-249. doi:10.1016/S2213-8587 (13)70086-X

62. Yoshihara A, Noh J, Yamaguchi T, et al. Treatment of graves' disease with antithyroid drugs in the first trimester of pregnancy and the prevalence of congenital malformation. J Clin Endocrinol Metab. 2012;97(7):2396-2403. doi:10.1210/jc.2011-2860

63. Vestergaard P, Mosekilde L. Fractures in patients with hyperthyroidism and hypothyroidism: a nationwide follow-up study in 16,249 patients. Thyroid. 2002;12(5):411-419. doi:10.1089/1050725023202 88401

64. Lindsay TJ, Vitrikas KR. Evaluation and treatment of infertility. Am Fam Physician. 2015;91(5):308-314. 


\section{Supplementary materials}

Table SI Types of congenital malformations (CMs) in the children born by women with hypothyroidism from I January 2006 to June 2016, and followed until 30 June 2017

\begin{tabular}{|c|c|c|}
\hline Types of CMs according to ICD- $10^{a}$ & Exposed, $\mathbf{N}(\%)$ & Unexposed, N (\%) \\
\hline CMs within the nervous system [Q00-Q07] & $3(0.43)$ & $54(0.19)$ \\
\hline CMs within eye, ear, facem, and neck [Q।0-Q।8] & $\mathrm{I}(0.14)$ & I0I (0.36) \\
\hline CMs within circulation organs [Q20-Q28] & $45(6.45)$ & $939(3.31)$ \\
\hline CMs in the respiratory organs [Q30-Q34] & $6(0.86)$ & $94(0.33)$ \\
\hline Cleft lip and palate [Q35-Q37] & $4(0.57)$ & $71(0.25)$ \\
\hline Other CMs in the digestive organs [Q38-Q45] & $27(3.87)$ & $40 I(I .4 I)$ \\
\hline CMs in the genital organs $[\mathrm{Q} 50-\mathrm{Q} 56]^{\mathrm{b}}$ & $7(1.01)$ & $326(1.15)$ \\
\hline CMs in the urinary system [Q60-Q64] & $8(1.15)$ & II4 (0.4) \\
\hline $\mathrm{CMs}$ in the bones and muscles [Q65-Q79] ${ }^{\mathrm{C}}$ & $22(3.15)$ & $729(2.57)$ \\
\hline Other CMs [Q80-Q89] $]^{\mathrm{d}}$ & $4(0.57)$ & $84(0.3)$ \\
\hline Chromosome anomalies [Q90-Q99] & $3(0.43)$ & $50(0.18)$ \\
\hline
\end{tabular}

Notes: a ${ }^{2}$ Children with CMs might have more than one type of CMs. ${ }^{b}$ Exclusive Q5.5x undescended testis. ${ }^{\mathrm{C}}$ Exclusive Q65x dislocations of the hip. ${ }^{\mathrm{d}} \mathrm{No}$ distinct pattern of CMs within this group.

Table S2 Types of congenital malformations (CMs) in the children born by women with hyperthyroidism from I January 2006 to June 2016, and followed until 30 June 2017

\begin{tabular}{|c|c|c|}
\hline Types of CMs according to ICD- $10^{a}$ & Exposed, $\mathbf{N}(\%)$ & Unexposed, N (\%) \\
\hline CMs within the nervous system [Q00-Q07] & $4(0.57)$ & $50(0.18)$ \\
\hline CMs within eye, ear, face, and neck [Q।0-Q।8] & I (0.14) & $85(0.30)$ \\
\hline CMs within circulation organs [Q20-Q28] & $10(1.43)$ & $832(2.94)$ \\
\hline CMs in the respiratory organs [Q30-Q34] & $I(0.14)$ & $86(0.30)$ \\
\hline Cleft lip and palate [Q35-Q37] & $0(0)$ & $63(0.22)$ \\
\hline Other CMs in the digestive organs [Q38-Q45] & $3(0.43)$ & $327(1.15)$ \\
\hline CMs in the genital organs $[\mathrm{Q} 50-\mathrm{Q} 56]^{\mathrm{b}}$ & $5(0.72)$ & $287(1.01)$ \\
\hline CMs in the urinary system [Q60-Q64] & $9(1.29)$ & $96(0.34)$ \\
\hline $\mathrm{CMs}$ in the bones and muscles [Q65-Q79] ${ }^{\mathrm{C}}$ & $8(1.15)$ & $624(2.20)$ \\
\hline Other CMs [Q80-Q89] ${ }^{\mathrm{d}}$ & $I(0.14)$ & $74(0.26)$ \\
\hline Chromosome anomalies [Q90-Q99] & $0(0)$ & $39(0.14)$ \\
\hline
\end{tabular}

Notes: a Children with CMs might have more than one type of CMs. ${ }^{b}$ Exclusive Q5.5x undescended testis. ${ }^{\circ}$ Exclusive Q65x dislocations of the hip. ${ }^{d}$ No distinct pattern of CMs within this group.

\section{Publish your work in this journal}

Clinical Epidemiology is an international, peer-reviewed, open access, online journal focusing on disease and drug epidemiology, identification of risk factors and screening procedures to develop optimal preventative initiatives and programs. Specific topics include: diagnosis, prognosis, treatment, screening, prevention, risk factor modification, systematic reviews, risk \& safety of medical interventions, epidemiology \& biostatistical methods, and evaluation of guidelines, translational medicine, health policies \& economic evaluations. The manuscript management system is completely online and includes a very quick and fair peer-review system, which is all easy to use. 